Contract No. and Disclaimer:

This manuscript has been authored by Savannah River Nuclear Solutions, LLC under Contract No. DE-AC09-08SR22470 with the U.S. Department of Energy. The United States Government retains and the publisher, by accepting this article for publication, acknowledges that the United States Government retains a non-exclusive, paid-up, irrevocable, worldwide license to publish or reproduce the published form of this work, or allow others to do so, for United States Government purposes. 


\title{
Characterization of Pd Impurities and Finite-Sized Defects in Detector Grade CdZnTe
}

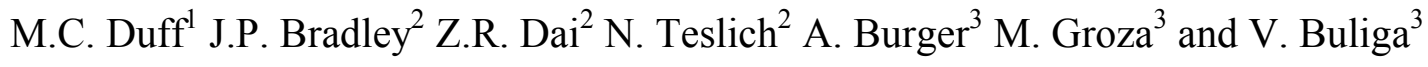 \\ ${ }^{1}$ Savannah River National Laboratory, Aiken, SC 29808, U.S.A. \\ ${ }^{2}$ Lawrence Livermore National Laboratory, Livermore, CA 94550, U.S.A. \\ ${ }^{3}$ Fisk University, Nashville, TN 37208, U.S.A.
}

\begin{abstract}
Synthetic CdZnTe or "CZT" crystals are highly suitable for $\gamma$-spectrometers operating at the room temperature. Secondary phases (SP) in CZT are known to inhibit detector performance, particularly when they are present in large numbers or dimensions. These SP may exist as voids or composites of non-cubic phase metallic Te layers with bodies of polycrystalline and amorphous CZT material and voids. Defects associated with crystal twining may also influence detector performance in CZT. Using transmission electron microscopy, we identify two types of defects that are on the nano scale. The first defect consists of $40 \mathrm{~nm}$ diameter metallic $\mathrm{Pd} / \mathrm{Te}$ bodies on the grain boundaries of Te-rich composites. Although the nano-Pd/Te bodies around these composites may be unique to the growth source of this CZT material, noble metal impurities like these may contribute to SP formation in CZT. The second defect type consists of atom-scale grain boundary dislocations. Specifically, these involve inclined "finite-sized" planar defects or interfaces between layers of atoms that are associated with twins. Finite-sized twins may be responsible for the subtle but observable striations that can be seen with optical birefringence imaging and synchrotron X-ray topographic imaging.
\end{abstract}

\section{INTRODUCTION}

Synthetic $\mathrm{Cd}_{1-\mathrm{x}} \mathrm{Zn}_{\mathrm{x}} \mathrm{Te}$ or "CZT" crystals are candidate materials for use as a room temperature-based radiation spectrometer. The ternary alloy with $10 \% \mathrm{Zn}, \mathrm{Cd}_{0.9} \mathrm{Zn}_{0.1}$ Te has a high band gap of $\sim 1.6 \mathrm{eV}$ and high resistivity form $\left(\sim 10^{10} \Omega \bullet \mathrm{cm}\right)$, which facilitates its use as a simplistic semiconductor spectrometer and imager that requires little power and no cryogenic cooling for operation. Over the last decade, the methods for growing high quality CZT have improved the quality of the produced crystals however various defects remain in these materials that can influence their performance as radiation spectrometers. For example, various structural heterogeneities within the crystals, such as twinning, pipes, grain boundaries (polycrystallinity), elemental impurities and secondary phases (SP) can have an impact on the detector performance $[1,2,3,4]$.

Transmission electron microscopy (HR-TEM) has been used to examine the atom scale defects in SP and the bulk of CZT [1,5,6,7]. The morphology and crystalline plane orientation of the SP that consisted of dendritic and Te-rich material in CZT have been investigated in a highly detailed study using scanning electron microscopy (SEM) and electron backscatter diffraction (EBSD) [8]. We recently characterized SP in a sample of modified vertical Bridgman grown CZT from Yinnel Tech (YT). This material possessed properties that are suitable for its use in $\gamma$ - 
radiation detectors. Our study, which used SEM and HR-TEM techniques identified mostly empty $20 \mu \mathrm{m}$ SP voids in addition to some similarly sized Te-rich SP are published in Duff et al. (2009) [9].

The reason for the formation of the Te-rich SP could not be determined from our study. Elemental impurities in the melt may contribute to their formation. Information on elemental composition of bulk impurities (usually performed by glow discharge mass spectrometry) is often available for CZT materials but information on the composition of such impurities within SP is fairly limited [e.g., ref.10]. The type of growth method used may influence SP formation as well.

Our present study continues with the characterization of this Te-rich material as well as bulk material from the same wafer of material. It discusses the elemental composition and morphology of the impurities that were isolated from the Te-rich SP, which occupied $\sim 10 \%$ of the SP in this particular CZT material. It also presents data on the atom scale defects that were observed in the bulk of this material.

\section{EXPERIMENTAL DETAILS}

A high performance radiation spectrometer CZT crystal (378) material (received as a $12.1 \mathrm{x}$ $11.3 \times 6.8 \mathrm{~mm}^{3}$ material) was grown according to the Modified Vertical Bridgman (MVB) to have $\mathrm{x}=0.1 \mathrm{Zn}$ content as in Li et al. procured from YT, South Bend, IN [11]. This type of meltbased growth involves the movement of crucible containing a high purity (typically $>7 \mathrm{~N}$ to $8 \mathrm{~N}$ ) polycrystalline $\mathrm{CdZnTe}$ charge through a high temperature furnace. The preparation of this CZT material in this study is described in Duff et al. (2007; 2009) [9,12]. The CZT (as the subsampled 378-3 portion) was provided to LLNL for examination by SEM using an FEI Nova 600 Nanolab Dualbeam focused ion beam scanning electron microscope (FIB-SEM) at $5 \mathrm{keV}$ to analyze and produce thin sections of SP-based areas of interest (through ion-beam milling) for HR-TEM studies [as described in ref. 9] as shown in fig. 1.

The HR-TEM studies were performed with a $200 \mathrm{keV}$ FEI Technai20 G2 FEG monochromated scanning transmission electron microscope (STEM) with high angle annular dark field (HAADF) detector using a $\mathrm{Si}(\mathrm{Li})$ solid state X-ray detector with 0.3 steradians solid angle. For our TEM studies, two thin sections (shown in figs. 2 and 3) from our prior TEM study [9] were used in the current study. However, one of the sections (specifically, the one made from the bottom portion of a SP negative crystal or void, as shown in fig. 2) had to be thinned for our examination of atom scale defects within the surrounding bulk.

Detector performance and methodology information for 378 are also published [12]. Optical birefringence imaging performed with this YT material using $1150 \mathrm{~nm}$ IR illumination. The Optical birefringence imaging that we used for our CZT characterization with this and other CZT material are published elsewhere as well [13]. 

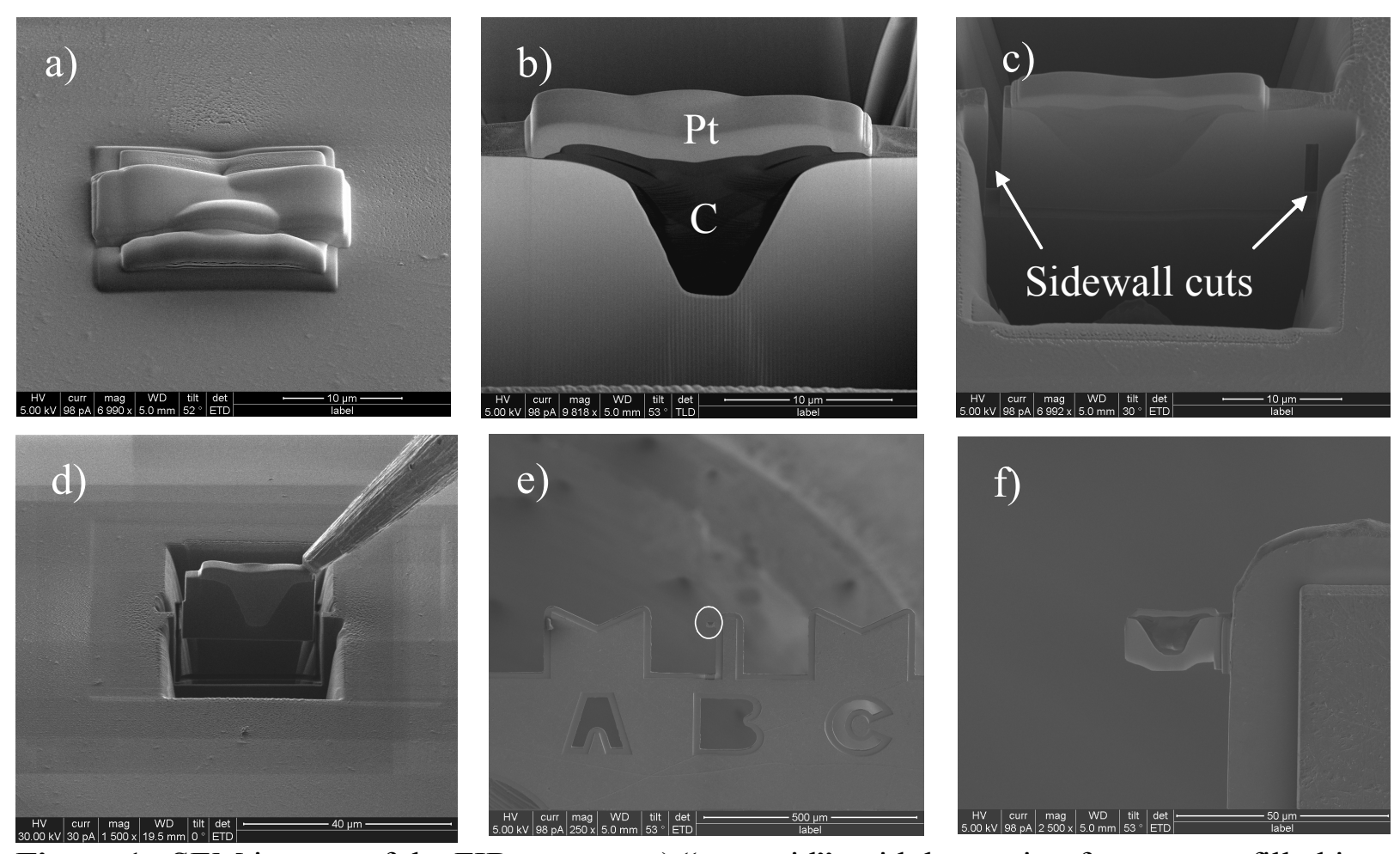

Figure 1. SEM images of the FIB process: a) "pyramid" void depression feature was filled in with carbon (C) using the in-situ deposition capabilities of the FIB. The surface of the feature was covered with platinum $(\mathrm{Pt})$. Carbon and $\mathrm{Pt}$ layers reduce the effects of potential beam damage during the FIB milling process. In b), the feature after the ion beam has trenched either side of the Pt strap - revealing a cross-sectional sample view. In c), the section is further thinned to $\sim 1 \mu \mathrm{m}$ and the ion beam is used to make sidewall and under-cuts to enable the extraction of the section. In d), the in-situ extraction of the section from the bulk. In e) post attachment of the section to the TEM grid and f) after further ion thinning which produced a $\sim 100 \mathrm{~nm}$ section.

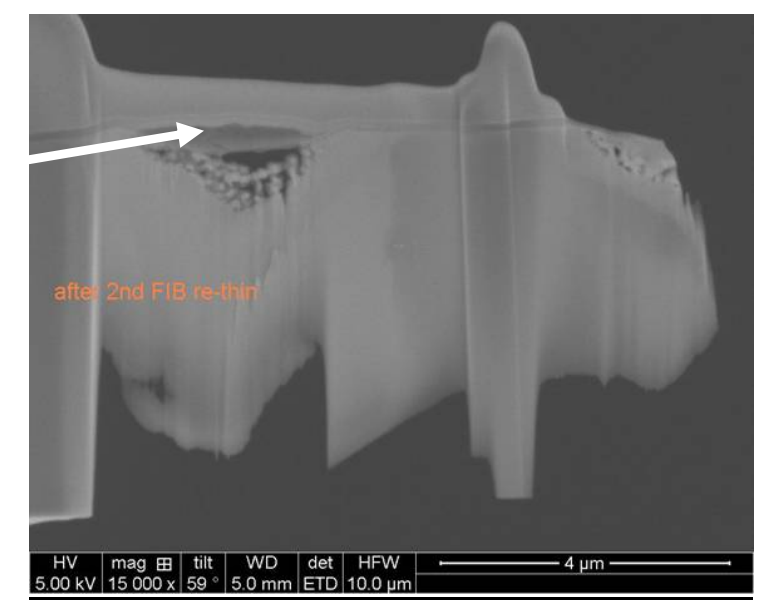

Figure 2. Re-thinning and re-polishing of a second thin section of CZT 378-3 for higher resolution imaging in this study (initial FIB preparation shown in fig. 1). This thin section was isolated in Duff et al. (2009) and was made of bulk single crystal material that was proximal to a void containing nanoparticulate $(\mathrm{Cd}, \mathrm{Te}, \mathrm{Zn}$, and $\mathrm{Si}$ ) residue (see arrow near remainder of void). 


\section{RESULTS}

\section{Characterization of SP impurities}

In our prior studies with 378-3, we identified several 20- $\mu$ m sized hexagonal-shaped entities that were filled with metallic Te (like that shown in fig. 3) on the Te- and Cd-rich faces [9]. In our current study, a closer examination of the composition and crystalline properties of the filled portions of these heterogeneities (SP) revealed the existence of polycrystalline (PC) Te metal, amorphous (AM) CZT and PC CZT. Within the PC Te metal, we observed $40 \mathrm{~nm}$ particles that were located at grain boundaries within the Te-rich material as shown in fig. 3a-f and fig. $4 a$.

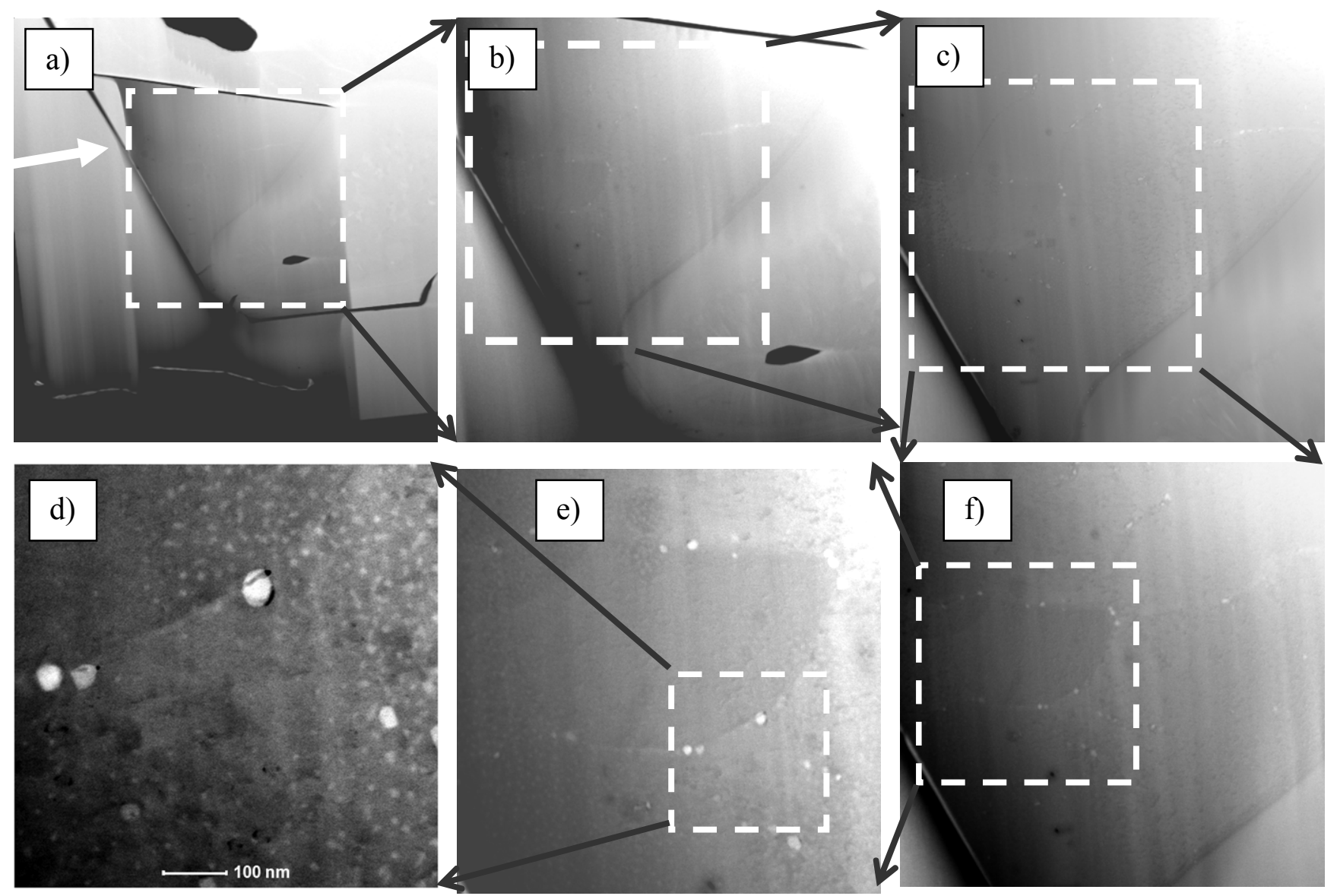

Figure 3. SEM images of the thin section of the Te-rich SP in 378-3 that was later examined using TEM. The teardrop-shaped object (in center right of a) contains a void-like cave. The teardrop shaped object is bordered by polycrystalline (PC) CdZnTe that has an amorphous (AM) $\mathrm{CdZnTe}$ rim. The material outside of the AM CdZnTe rim is PC Te. The white arrow in a) points to the boundary between the SP and the bulk single crystal CZT. Images revealing the presence of a nano-size object within the AM Te material in the SP are shown in a) through f). Closer examination of the nano-sized object indicates that some are oriented along a grain boundary inside the metallic PC Te of the Te-rich SP. [Note: Image adapted from fig. 4 in ref. 9 and relative to fig. 4 in ref 9, the images a) and f) are reversed from left to right.] 

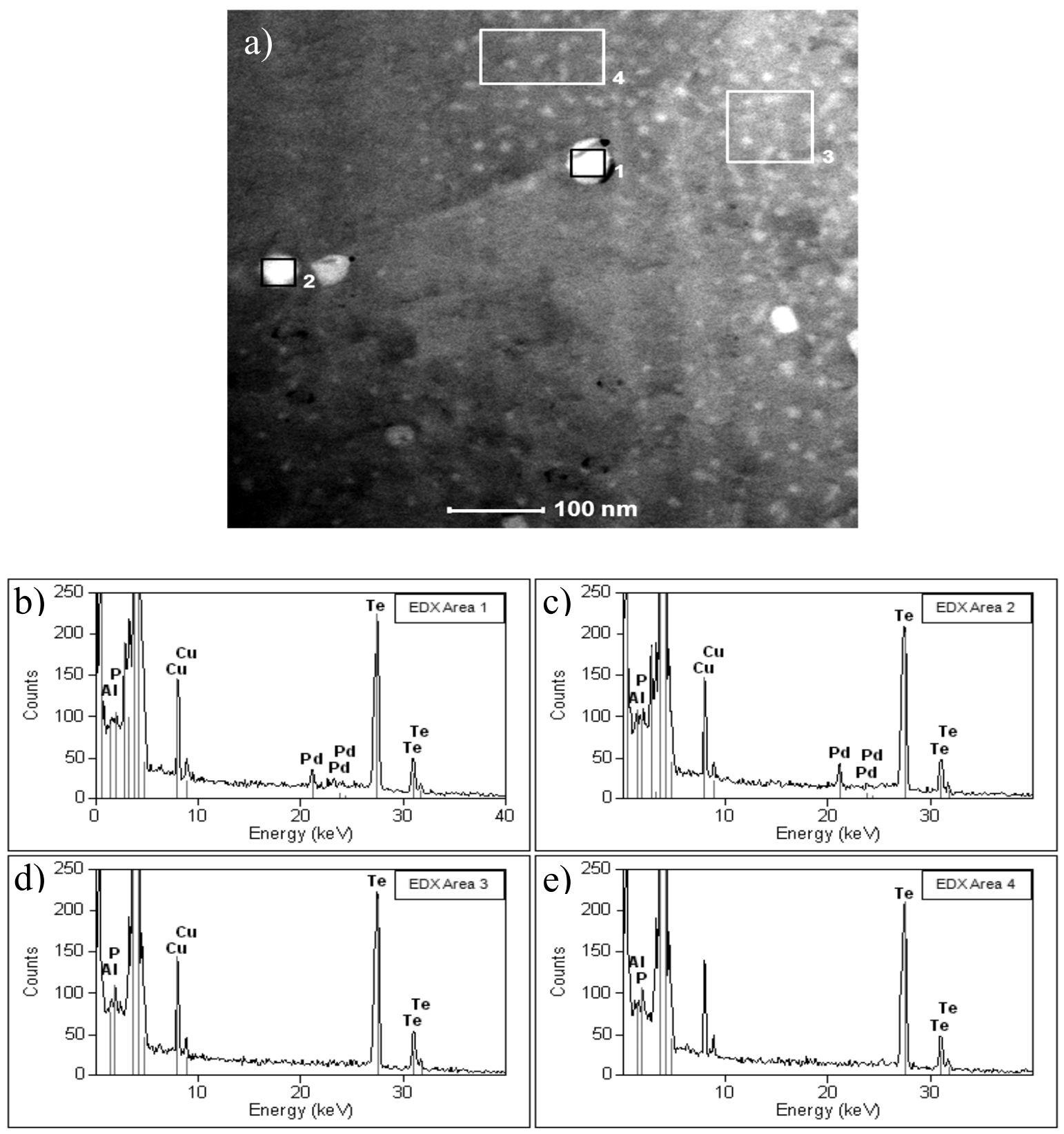

Figure 4. Elemental identification of four various regions in the PC Te metal portion of the Terich phase shown in a). The white areas in a) correspond to $40 \mathrm{~nm}$-sized nano particles of PdTe as shown in b) and c). Bulk material analyses by EDX reveal the absence of Pd and a Te-rich material (PC Te metal, according to ref. 9).

These particles were primarily Pd-rich but they were also rich in Te as evidenced by the energy dispersive X-ray (EDX) analyses (shown in fig. 4b-e). Trace levels of Al and $\mathrm{P}$ were also found throughout the material regardless of location as shown in fig. 4b-e. 


\section{Characterization of line-type defects}

The optical birefringence imaging studies with sample 378 revealed little differences in the images that were taken without the cross analyzer as shown in fig. 5 (images in left box). However, several features can be observed in the images taken with the cross analyzer in fig. 5 (see images in box at right).

Several striations appear vertically and at angles throughout the material may indicate the presence of non-uniformity in the electric field. This non-uniformity could potentially impact charge transport and have a detrimental effect on radiation detector performance - although we know that this material is detector grade. Striations have been observed with optical birefringence images in highly resistive detector grade CZT material grown using the traveling heater method by Redlen Technologies (British Columbia, Canada) [13].

We anticipate that these striae represent a certain measurable level of crystalline strain in the material. This level of strain may be induced at the atom level. We have observed subtle striations (or waviness) in CZT using synchrotron X-ray topography imaging studies with material grown from the same boule as well as from YT samples 378 and YT-5 [9,14]. These studies indicate that crystalline strain exists based on the differences in the long range order can be observed using this sensitive diffraction-based imaging technique.

We performed a closer examination at the atomic level to study what may be producing the striations. We note that these striations are not apparent through our visual examinations of the material or transmission-based IR imaging (see data with polarizer in fig. 5 left box and without polarizer; and IR transmission image in fig. 1 in ref. 9). These methods can often reveal the presence of gross defects such as twining or other grain boundaries within the single phase.

Our HR-TEM studies with HAADF imaging reveal the presence of what at first appears to be a dissociated dislocation, but closer examination reveals otherwise. This type of dislocation could be expected to form a stacking fault. However, the number of planes on both sides of the defect is conserved. This feature appears to be a type of inclined defect since, as focus in the HAADF image is changed, the defect migrates in a direction perpendicular to the lattice fringes. The structural nature and abundance of these defects in CZT is not yet known and we are investigating the possibility that they are related to the striae shown in fig. 5. 

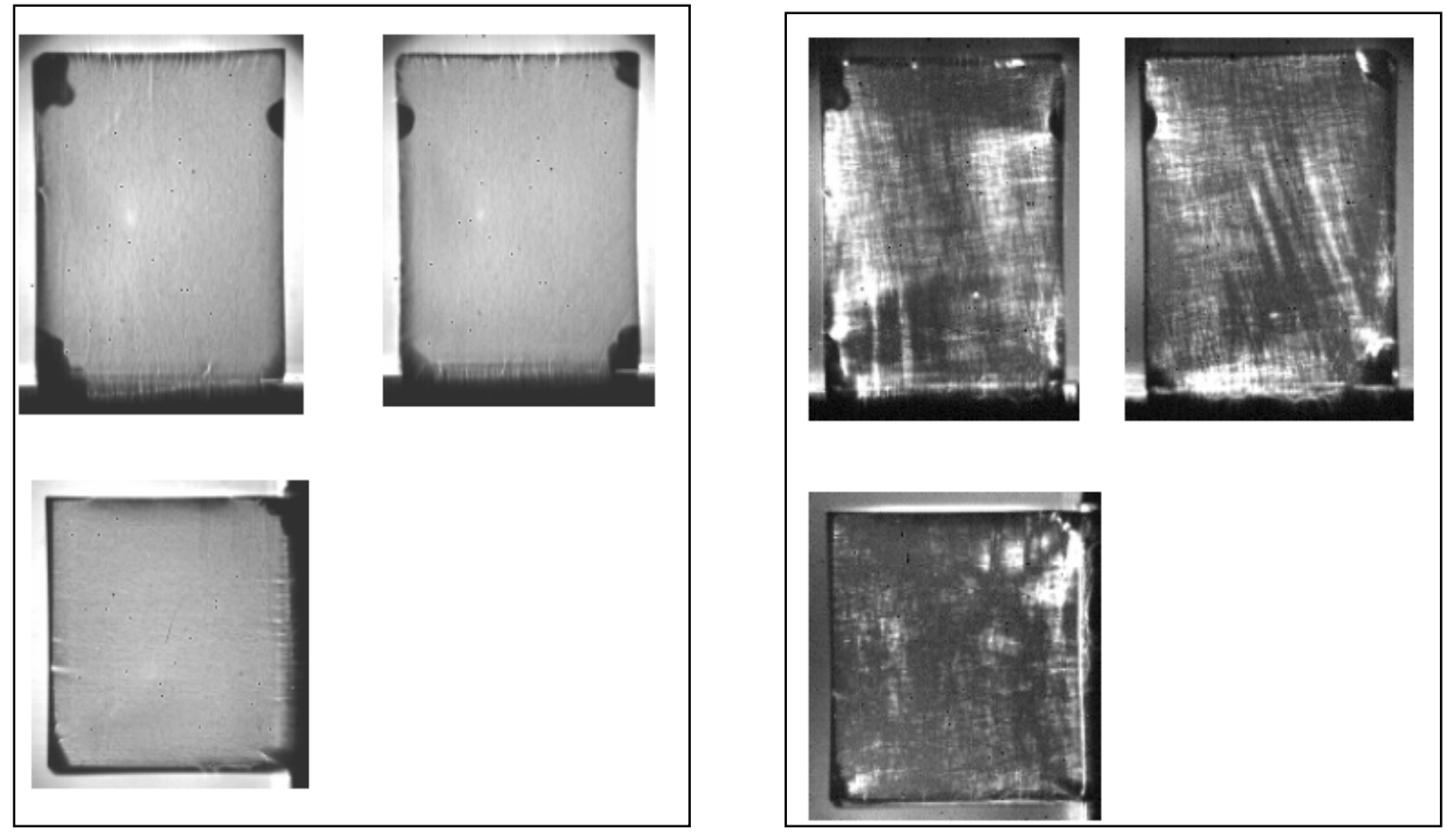

Figure 5. Cross polarized IR images of the same three perpendicular sides of as received YT 378 $\left(12.1 \times 11.3 \times 6.8 \mathrm{~mm}^{3}\right)$ material reveal for $1150 \mathrm{~nm}$ polarized light with only the polarizer at 45 degrees with vertical (images in box at left) and the polarizer with cross analyzer at 45 degrees (images in box at right).
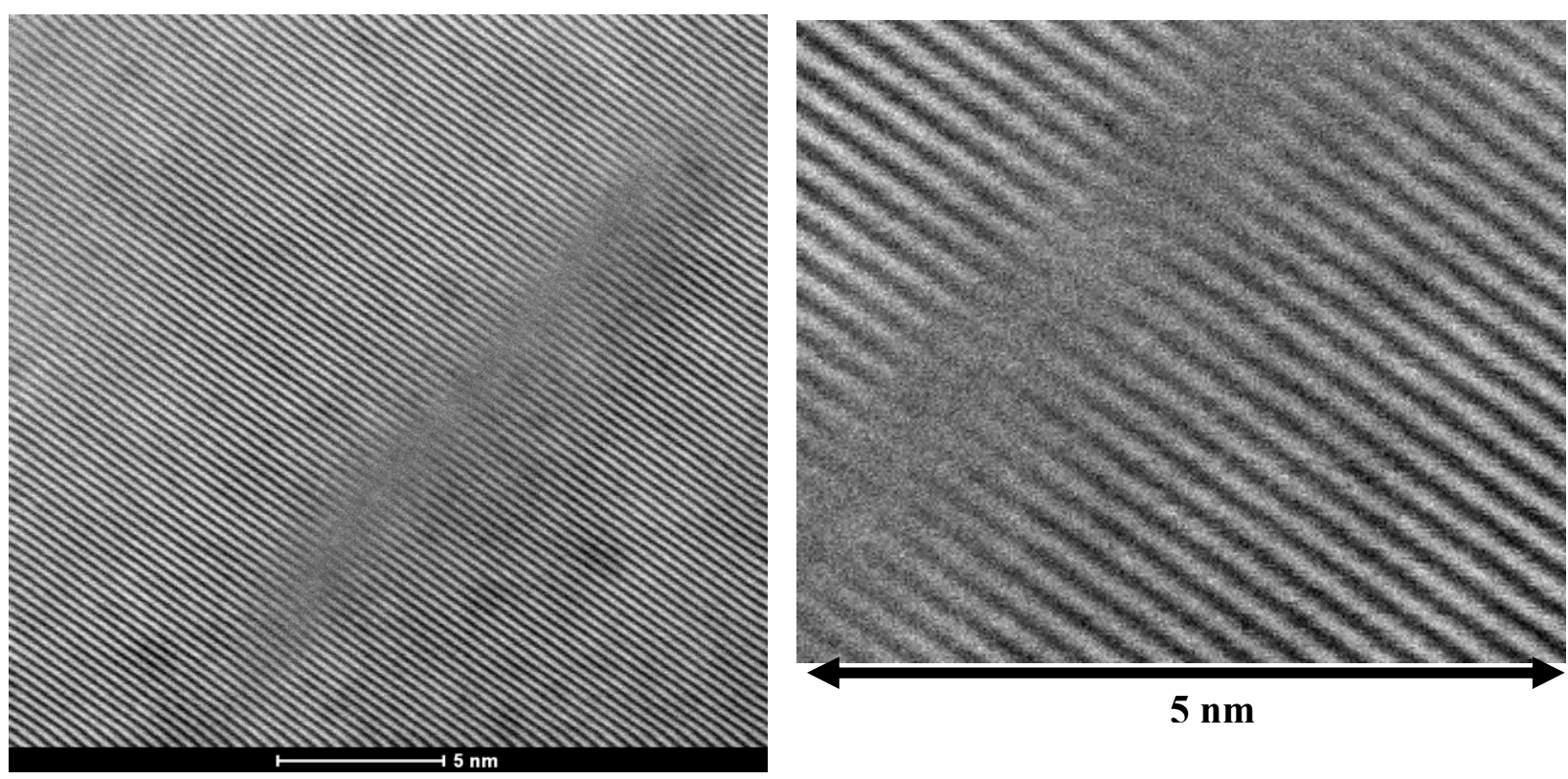

Figure 6. HAADF imaging of atom scale defect in CZT 378-3 from a single crystal area near the void with left) the parent image and right) an inset from a) with $3 \mathrm{x}$ magnification. 


\section{DISCUSSION}

\section{$\underline{\text { SP impurities }}$}

The melt is often Te-rich and it is conceivable that impurity elements such as Pd could be expected to form one or more compounds with the excess Te. Metallic Pd is generally a face centered cubic (fcc) material [15]. Metallic Pd will form solid solutions with Te but these phases are not (primarily fcc) [16]. Examples of phases that will form below the temperature of cubic CdZnTe growth $\left(\sim 1100\right.$ deg $\mathrm{C}$ for MVB) include but are not limited to: $\mathrm{Pd}_{3} \mathrm{Te}$ (body centered cubic or bcc), $\mathrm{Pd}_{20} \mathrm{Te}_{7}$ (rhombohedral) and $\mathrm{Pd}_{8} \mathrm{Te}_{3}$ (orthorhombic) and mixtures thereof (forming between 754 and $780 \mathrm{deg}$. C). Although the starting material for the CZT seed charge may be of high purity, several impurities can be expected to be insoluble in the melt. These impurity phases may also provide locations for other phases that are unable to dissolve within the solid single phase structure due to their composition or degree (or type) of crystallinity. This is evidenced by the conglomeration of the various AM and PC CdZnTe and Te phases that are present in the area that the nano-phase PdTe is found. Other features that can be observed include micro-twining and grain boundary-type features (such near the large cave as in fig. 3a).

Detectable impurities containing $<0.5 \mu \mathrm{m}$ Au particles and larger microphases of alumina have been observed within SP or in the case of calcium carbonate $\left(\mathrm{CaCO}_{3}\right)$ within the entirety of the SP in prior studies with MVB-grown CZT material from WSU $[10,17]$. We have observed nanoparticulate $\mathrm{Cd} / \mathrm{Zn} / \mathrm{Te} / \mathrm{Si}$ residue in $\mathrm{SP}$ voids in $\mathrm{YT} 378$. It is reasonable to expect that impurities will assimilate in some manner when they are not soluble in the single phase. It is also reasonable that these impurities may reside within another material (like PC Te) that offers more stability than that of the single phase.

We do not find the PC Te in the Te-rich SP to be an artifact of the electron irradiation. Also, the Pd that we observe is not an artifact of the sample preparation because Pd is not used in the electron microscopes that were used for the sample preparation or analyses.

\section{$\underline{\text { Line-type defects }}$}

Marquis et al. $(2004,2007)$ observed similar "finite-sized" grain boundary defects with $\{112\}$ twins that formed junctions with $\{111\}$ twins in gold [as observed in fig. 1 of ref. 18 and fig. 3 of ref. 19]. These examples of offset planes for fcc gold metal are similar to what we have observed with CZT, which is possesses which can be referred to as fcc (i.e., with two offset fcc lattices). Although of interest but not performed in our study, first principles calculations, could yield information about the total energy of the boundary per unit length and level of strain that is compensated for in this material (in ref. 18).

On a larger scale, the inclined planar defects that are observed at the atom scale may propagate enough lattice strain to be visible through techniques (e.g., as striae as viewed with optical birefringence imaging and a waviness appearance in X-ray topography) and that are highly sensitive to strain but to not exhibit enough strain to cause large scale grain boundaries that are highly visible with less sensitive methods as previously discussed.

We noted that our study findings differ from those of Zeng et al. (2009), who examined abrupt, large-scale twin boundaries and sub-boundary networks with bulk CZT grown using the MVB method with HR-TEM. Our observations examined differences on the atom scale rather than that on the larger 200 to $1000 \mathrm{~nm}$ scale. 


\section{CONCLUSIONS}

This study presents new observations about the morphology and composition of the nanosized defects in detector grade CZT. We find although Pd is used as a coating for many electron microscopy analyses (in other electron microscopes), the Te-rich Pd nano particles that we observe are not the results of $\mathrm{Pd}$ that has been used for this purpose.

\section{ACKNOWLEDGMENTS}

Work supported by US DOE - National Nuclear Security Administration, through the Office of Nonproliferation and Verification Research and Development (NA-22) and National Science Foundation through the Fisk University Center for Physics and Chemistry of Materials (CPCoM), Cooperative Agreement CA: HRD-0420516 (CREST program) and through and from US DOE NA-22 Grant No. DE-FG52-05NA27035. We thank Dr. Doug Medlin (of LLNL) for his helpful comments. 


\section{REFERENCES}

1. J. R. Heffelfinger, D. L. Medlin, and R. B. James. MRS Symp. Ser. 487, 49-54 (1998).

2. M. Schieber, T. E. Schlesinger, R. B. James, H. Hermon, H. Yoon, and M. Goorsky. J. Cryst. Growth 237-239, 2082 (2002).

3. C. Szeles and M. C. Driver. SPIE Proc. 3446, 1 (1998).

4. J. Shen, D. K. Aidun, L. Regel, and W. R. Wilcox. Cryst. Growth 132, 250-260 (1993).

5. T. Wang, W. Jie, and D. Zeng. Mater. Sci. Engin. A 472, 227-230 (2008).

6. S. Rai, S. Mahajan, S. McDevitt, and C. J. Johnson. J. Vac. Sci. Tech. B9, 1892 (1996).

7. D. Zeng, W. Jie, T. Wang, and H. Zhou. J. Crystal Growth 311, 4414-4417 (2009).

8. C. Henager, D. J. Edwards, A. L. Schemer-Kohrn, M. Bliss, and J. E. Jaffe. J. Crystal Growth 312, 507-513 (2010).

9. M. C. Duff, D. B. Hunter, A. Burger, M. Groza, V. Buliga, J. P. Bradley, G. Graham, Z. R. Dai, N. Teslich, D. R. Black, and A. Lanzirotti. J. Mater. Res. 24, 1361-1367 (2009).

10. M. C. Duff, K. G. Lynn, K. Jones, Z. R. Dai, J. P. Bradley, and N. Teslich. SPIE Proc. 7449 $74490 \mathrm{~N}$ (2009) (oral and written publications).

11. L. Li, F. Lu, C. Lee, G. Wright, D. R. Rhiger, S. Sen, K. S. Shah, M. R. Squillante, L. Cirinano, R. B. James, A. Burger, P. Luke, and R. Olson. SPIE Proc. 4784, 76 (2003).

12. M. C. Duff, D. B. Hunter, P. Nuessle, D. R. Black, H. Burdette, J. Woicik, A. Burger, and M. Groza. J. Elect. Mater. 36, 1092-1097 (2007).

13. S. A. Awadalla, J. Mackenzie, H. Chen, B. Redden, G. Bindley, M. C. Duff, A. Burger, M. Groza, V. Buliga, J. P. Bradley, Z. R. Dai, N. Teslich, and D. R. Black. J. Crystal Growth 312, 507-513 (2010).

14. M. C. Duff, D. B. Hunter, A. Burger, M. Groza, V. Buliga, and D. R. Black. Appl. Surf. Sci. 254, 2889-2892 (2008).

15. M. Harada, K. Asakura, Y. Ueki, and N. Toshima. J. Phys. Chem. 96, 9730-9738. (1992).

16. H. Okamoto. J. Phase Equilibria 13, 73-79 (1992).

17. M. C. Duff, K. G. Lynn, K. Jones, R. Soundararajan, J. P. Bradley, H. Ishii, J. Aguiar, and P. Wozniakiewicz. SPIE Proc. 7805, 74490N (2010).

18. E. A. Marquis, J. C. Hamilton, D. L. Medlin, and F. Leonard. Phys. Rev. Lett. 93, 1-4 (2004).

19. E. A. Marquis, D. L. Medlin, and F. Leonard. Acta Materialia 55, 5917-5923 (2007). 